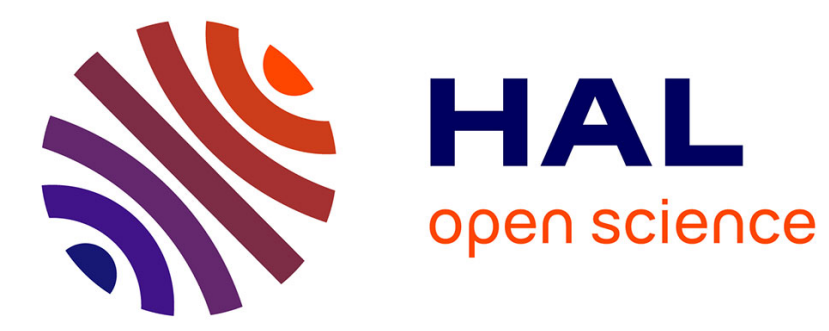

\title{
Nonlinear control of a DC MicroGrid for the integration of distributed generation based on different time scales
}

\author{
Sabah Benamane Siad, Ahmad Malkawi, Gilney Damm, Luiz Lopes, Lilia
}

Galai-Dol

\section{- To cite this version:}

Sabah Benamane Siad, Ahmad Malkawi, Gilney Damm, Luiz Lopes, Lilia Galai-Dol. Nonlinear control of a DC MicroGrid for the integration of distributed generation based on different time scales. International Journal of Electrical Power \& Energy Systems, 2019, 111, pp.93-100. 10.1016/j.ijepes.2019.03.073 . hal-02090612

\section{HAL Id: hal-02090612 \\ https://hal.science/hal-02090612}

Submitted on 4 Apr 2019

HAL is a multi-disciplinary open access archive for the deposit and dissemination of scientific research documents, whether they are published or not. The documents may come from teaching and research institutions in France or abroad, or from public or private research centers.
L'archive ouverte pluridisciplinaire HAL, est destinée au dépôt et à la diffusion de documents scientifiques de niveau recherche, publiés ou non, émanant des établissements d'enseignement et de recherche français ou étrangers, des laboratoires publics ou privés. 


\title{
Nonlinear control of a DC MicroGrid for the integration of distributed generation based on different time scales
}

\author{
Sabah B Siad, Paris Saclay University, Ahmad Malkawi, University of Jordan, \\ Gilney Damm, Paris Saclay University, Luiz Lopes, Concordia University, \\ and Lilia Galai Dol, Efficacity institute
}

\begin{abstract}
This paper is a contribution for DC MicroGrid control and introduces a rigorous dynamics' analysis, taking into account intermittency effects. We propose an hierarchical control scheme, based on nonlinear control theory, in particular Lyapunov, backstepping and input/output feedback linearization. The resulting algorithms are simple, comparable to standard nested PIs, and do not need important computer resources, such to be easily implemented in small micro-controllers. In addition to allowing an explicit stability analysis, they present the advantage of preserving performance for the whole operation region, and to be easy to tune. The proposed DC MicroGrid and its control are then verified either by computer simulations and by experiments. The results show the good performance of the system under variations on production and on consumption. Finally, the proposed scheme is compared to standard PI based controls, with better performance with simpler tuning procedure.
\end{abstract}

Index Terms-Power generation control, Nonlinear control systems, Power system control, Energy storage, Microgrids, DC Grids, Stability, Lyapunov methods.

\section{INTRODUCTION}

D UE to their intermittent nature, the large penetration of Distributed Generation (DG) can cause disturbances in the utility grid. To guarantee high quality electricity in this framework, it is interesting to produce and consume this distributed power close by, assuring locally the power balance with storage units, and then MicroGrids can be a good solution to attain this goal. The MicroGrid requires a suitable control strategy to provide a continuous and sufficient energy to load demand, balancing all power fluxes [1], [2], and it can be connected to the main network or not. In the present work a MicroGrid is used to integrate DG to time varying load, using storage system devices working in different time scales (battery and supercapacitor). This basic scheme indeed may represent rather complex grids since each element represents the aggregation of similar elements, and in particular the load

Sabah Benamane. Siad and Gilney Damm are with IBISC Paris Saclay University Paris, France. Email: sabah.siad-benamane@ibisc.fr and gilney.damm@ibisc.fr Ahmad Malkawi with University of Jordan,Amman Jordan Email:ah.malkawi@ju.edu.jo, Luiz Lopes with Concordia University, Montreal Canada Email: lalopes@encs.concordia.ca. Lilia Galai Dol with Efficacity institute Champs-sur-Marne , France Email:I.galai-dol@efficacity.com . can also aggregate the interconnection to the main grid [3]. The concept of MicroGrid integrating DG and storage can be the answer of interconnection constraints, and an efficient way to bring such power to the main grid [4], as well as providing ancillary services as voltage, frequency and inertia support. The power flow management and a rather complex control strategy become mandatory for such systems, and in the present case is constituted by an hierarchical control scheme. Although several control schemes are already applied to MicroGrids, as explained in the following they have hard limits when dealing with strong varying operating conditions; they usually not allow a formal stability proof, that leads to formal estimations of operation regions; they are very hard to tune, usually relying on exhaustive try and error by simulations to find control gains. The proposed control methods can deal with such drawbacks, and are much easier to tune than those found in the literature. Finally, they are expected to be easier to replicate in a distributed and interconnected way. In future works, it will be studied the interconnection of several similar MicroGrids in a systematic way.

Since several sources, storage systems and loads are natively $\mathrm{DC}$, in order to reduce power losses and to guarantee a better power quality we will use a DC MicroGrid. The challenge is to design a control structure (either for the whole grid than for each power converter) in such way that the interconnection of the DG to the network does not disturb the main grid, and to assure stability of the whole system under variation of production and consumption. The usual approach for controlling MicroGrids is linear control due its simplicity. In addition, it is widely known in both industry and academia, either in frequency domain via transfer functions as in state space, where nested PIDs, and sometimes robust techniques as LQR are the most used strategies. For example, the minor loop gain is a linear controller presented in [5] that relates source and load impedance's to determine stability in the grid. This controller has the advantage of maintaining system dynamics even when connecting more devices like filters. This impedance-based approach is also well fit when using state space modeling [6], [7].

Nevertheless, linear control in general is based on a linearized model, designed for only one operating point. This may represent an important inconvenient for the integration of varying sources like renewables, applied to also varying 
loads [8], [9], [10]. In addition to overcome limitations of linear theory, the real systems are split in several sub-systems with dedicated controllers for each. The final structure includes nested controllers, where the effects of one controller and its tuning on the others is never formally established. The system may then become very complex, and their tuning a real challenge. For all these reasons, nonlinear theory may provide a much broader approach that can cover all aspects, and provide rigorous analysis of the overall system for a well defined operation region. In addition, the final controllers are not much more complex than linear ones, and end up much simpler to tune, because their tuning comes from the Lyapunov function and the desired dynamics.

In the present work it is used System of Systems approach: the control is designed globally and accomplished locally (see [11] and [12]), also used in [13]. In the present case, the control structure of the DC MicroGrid is designed with two levels, the low level controller based on nonlinear control theory (Lyapunov, backstepping and input/output feedback linearization) assures exponential stability of each element to its own reference. On the top of that, high level controllers give references based on different objectives like power production optimization (MPPT); maximizing storage capacity; and in particular to accomplish power flow in all branches of the DC grid [14], [15]. The system's stability is formally analyzed by a constructive Lyapunov function, and assures exponential stability of the whole system under mild conditions, that in their turn define the operation conditions of the grid. The obtained system will then guarantee good dynamic performance and flexibility.

The theoretical development is then verified first by computer simulations, and then in an experimental setup. All tests show the good performance of the proposed control scheme for random variations on load and on generation. The proposed controller is also compared by computer simulations to the standard PI controllers. Even if PI can keep all variables inside their operation margins, the nonlinear controller shows a much better performance, and is much simpler to tune. Furthermore, in the nonlinear case it is well established its stability properties, the desired performance and the operation region.

\section{SYSTEM CONFIGURATION}

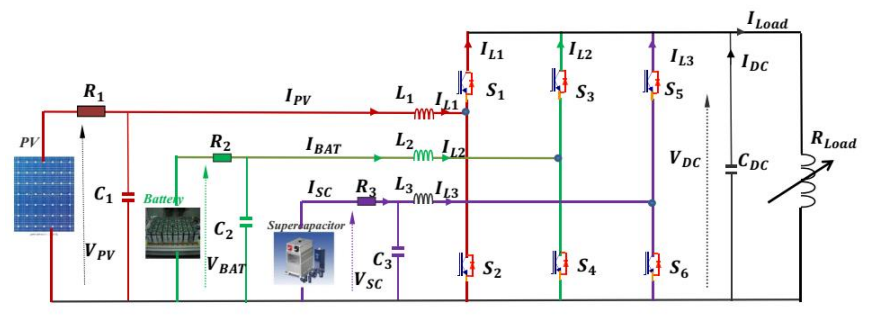

Figure 1: Three-Input $D C-D C$ Boost Converter Circuit

The structure of the DC MicroGrid is represented (to allow experiments in laboratory) by a simple scheme shown in figure (1). The PV array, battery and supercapacitor are connected to the DC MicroGrid by a Three-Input $D C-D C$ Boost Converter. One unidirectional leg for PV input representing the aggregation of all sources, two bidirectional legs for the storage elements, and the aggregation of all loads is connected to the DC MicroGrid.

\section{A. Model of Three-Input DC/DC Boost Converter}

This converter consists of a Three-Input DC/DC Boost Converter with six switches connected in parallel per phase. These switches are independently controlled with three different duty cycles. In the following it will be used these duty cycles to control the power flow among PV, storage and load.

The mathematical model is given based on power electronics averaging technique (see [16], [17] and [18]) controlled using Pulse Width Modulation (PWM). The state variables in this model are the capacitor voltages $V_{C 1}, V_{C 2}, V_{C 3}$, inductor currents $i_{L 1}, i_{L 2}, i_{L 3}$ and DC MicroGrid voltage $V_{D C}$.

$$
\begin{aligned}
\frac{d V_{C 1}}{d t} & =-\frac{1}{R_{1} C_{1}} V_{C 1}-\frac{1}{C_{1}} i_{L 1}+\frac{1}{R_{1} C_{1}} V_{P V} \\
\frac{d i_{L 1}}{d t} & =\frac{1}{L_{1}} V_{C 1}-\frac{\left[\left(R_{01}-R_{02}\right) u_{1}+R_{02}\right] i_{L 1}}{L_{1}}-\frac{\left(1-u_{1}\right)}{L_{1}} V_{D C} \\
\frac{d V_{C 2}}{d t} & =-\frac{1}{R_{2} C_{2}} V_{C 2}-\frac{1}{C_{2}} i_{L 2}+\frac{1}{R_{2} C_{2}} V_{B A T} \\
\frac{d i_{L 2}}{d t} & =\frac{1}{L_{2}} V_{C 2}-\frac{\left[\left(R_{03}-R_{04}\right) u_{2}+R_{04}\right] i_{L 2}}{L_{2}}-\frac{\left(1-u_{2}\right)}{L_{2}} V_{D C} \\
\frac{d V_{C 3}}{d t} & =-\frac{1}{R_{3} C_{3}} V_{C 3}-\frac{1}{C_{3}} i_{L 3}+\frac{1}{R_{3} C_{3}} V_{S C} \\
\frac{d i_{L 3}}{d t} & =\frac{1}{L_{3}} V_{C 3}-\frac{\left[\left(R_{05}-R_{06}\right) u_{3}+R_{06}\right] i_{L 3}}{L_{3}}-\frac{\left(1-u_{3}\right)}{L_{3}} V_{D C} \\
\frac{d V_{D C}}{d t} & =\frac{1}{C_{D C}}\left[\left(1-u_{1}\right) i_{L 1}+i_{S t o r a g e}-i_{L o a d}\right]
\end{aligned}
$$

where $R_{1}, R_{2}, R_{3}, L_{1}, L_{2}, L_{3}, C_{1}, C_{2}, C_{3}$ and $C_{D C}$ are known positive values of resistances, capacitors and inductors. For modeling the switches it was included small resistances ( $R_{01}, R_{02}, R_{03}, R_{04}, R_{05}$ and $R_{06}$ ) when they are driving, so the conduction losses are taken into account. $V_{P V}, V_{B A T}, V_{S C}$ and $V_{D C}$ are the photovoltaic panel, battery, supercapacitor and DC MicroGrid voltages respectively. The output measurement state is composed by $V_{C 1}, V_{C 2}, V_{C 3}, i_{L 1}, i_{L 2}, i_{L 3}$ and $V_{D C} \cdot u_{1}, u_{2}$ and $u_{3}$ will be the control inputs which define the duty cycles of the converter.

\section{Grid Control Strategy}

The objective of this work is to keep DC MicroGrid voltage in its nominal value and to assure stability of the whole system. To attain this goal, high level controllers provide references for the local ones. This higher level task is split in different objectives. The high level controller for the PV follows a MPPT algorithm. The high level controller for the storage system is given by a power flow management in DC MicroGrid. In this part, the DC MicroGrid voltage $V_{D C}$ is compared to its reference value $V_{D C}^{*}$, then it is computed the total current required to be provided/absorbed by the storage such as to fulfill the power flow and steer the DC grid voltage to its reference. We use a low-pass filter to split this current into two references. The average (low frequency) 
component of the signal is the reference for the battery current control. The dynamic (higher frequency) component becomes the reference for the supercapacitor current control.

The objective of this control strategy is to use each storage element in a different time scale; the role of the battery is to support slow transitory flow, while the supercapacitor supports fast transitory flows.

\section{A. Control Structure}

Figure (2) shows the block diagram representation of the MicroGrid control strategy. The objective of this algorithm is to stabilize DC MicroGrid voltage and to feed correctly load with desired power while reduce battery charge/discharge cycles.

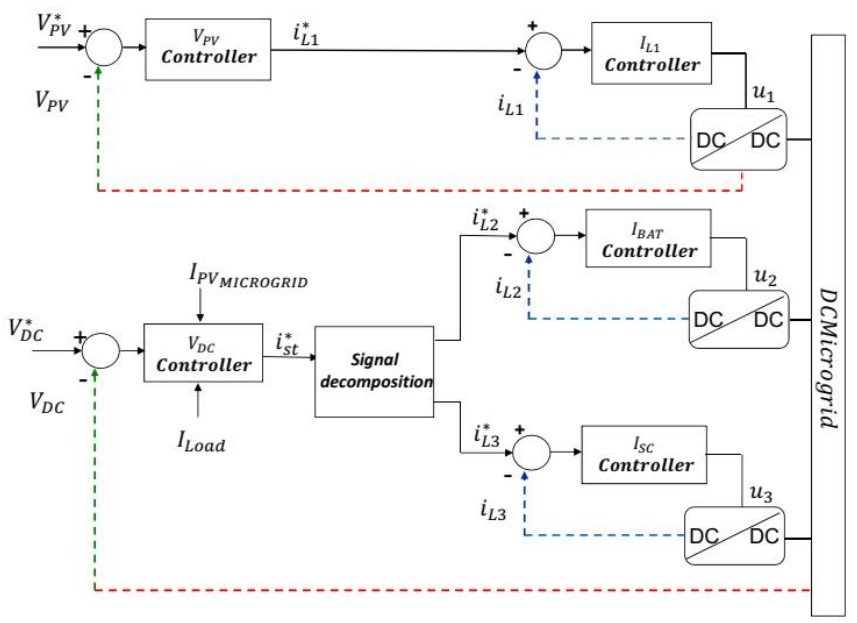

Figure 2: Structure Control

\section{B. High level controller}

1) High level controller for the Power Source : In order to maximize the solar panels producing capability, it is necessary to control its operating point to draw the maximum power from it. The algorithm of Maximum Power Point Tracking (MPPT) used in this work is an Incremental Conductance (IncCond) which delivers a reference voltage $V_{C 1}^{*}$ to be tracked by the lower level controller.

2) High level controller for the storage system: The proposed Hybrid storage consists of a Li-Ion Battery and a supercapacitor. The target of the control is to charge/discharge the storage component and to provide grid voltage stability during transients. The power needed to fulfill the power flow is represented by a current flowing from/to the storage to/from the DC-link capacitance.

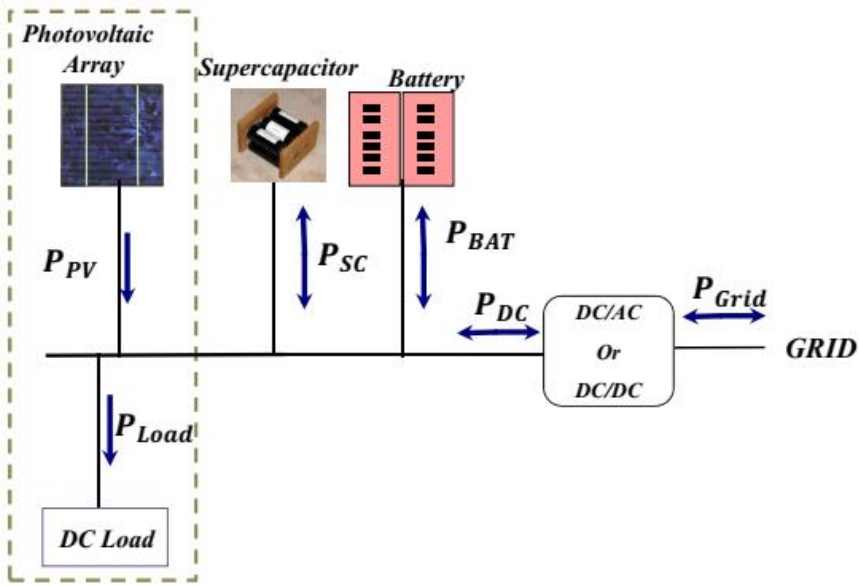

Figure 3: Power Flow

The power flow on the components in the proposed DC MicroGrid is shown in Figure (3) (see [19]). The sum of the output power of the photovoltaic array, and the storage is defined as follows:

$$
\begin{gathered}
P_{D C}=P_{P V}+P_{\text {Load }}+P_{S T} \\
P_{S T}=P_{B A T}+P_{S C}
\end{gathered}
$$

With $P_{P V}$ : PV power, $P_{S T}$ : Storage system power, $P_{B A T}$ : Battery power, $P_{S C}$ : Supercapacitor power, $P_{\text {Load }}$ Load power, $P_{D C}$ : DC grid power. From equation (2):

$$
C_{D C} V_{D C} \frac{d V_{D C}}{d t}=V_{D C}\left[i_{P V}+i_{\text {Storage }}-i_{\text {Load }}\right]
$$

which gives us the last equation of the system (1).

The reference value for $V_{D C}$ is the constant $V_{D C}^{*}$, a backstepping procedure is then adopted such as to design an $i_{S T}=i_{L 2}^{*}+i_{L 3}^{*}$ reference for $i_{L 2}$ and $i_{L 3}$ that will provide new desired dynamics for the DC bus voltage. In our study, the system is sized such that the battery and supercapacitor stay in $40 \%<S O C<80 \%$, so we work in the linear phase of the battery and supercapacitor.

\section{SyStem NONLINEAR CONTROL}

\section{A. Storage System Control Law}

1) Voltage Control (High Level Controller): The outer voltage loop is used to stabilize $V_{D C}$ by the following method. Lets first define the output tracking error:

$$
e_{V_{D C}}=\left(V_{D C}-V_{D C}^{*}\right)
$$

From system (1):

$$
\frac{d V_{D C}}{d t}=\frac{1}{C_{D C}}\left[\left(1-u_{1}\right) i_{L 1}+i_{\text {Storage }}-i_{\text {Load }}\right]
$$

and the desired dynamics of $V_{D C}$ as:

$$
\begin{aligned}
\dot{\alpha}_{7} & =K_{7}^{\alpha} e_{V_{D C}} \\
\dot{e}_{V_{D C}} & =-K_{7}\left(V_{D C}-V_{D C}^{*}\right)-\bar{K}_{7} \alpha_{7}
\end{aligned}
$$


Where $\alpha_{7}$ is now defined as an extended state (integral term). From (5) and (6) we can design:

$$
\begin{aligned}
i_{S T}^{*}= & -K_{7} C_{D C}\left(V_{D C}-V_{D C}^{*}\right)-\bar{K}_{7} C_{D C} \alpha_{7} \\
& -\left(1-u_{1}\right) i_{L 1}+\frac{V_{D C}}{R_{\text {Load }}}
\end{aligned}
$$

with $K_{7}, \bar{K}_{7}$ and $K_{7}^{\alpha}$, positive gains that are used to impose desired dynamics to the closed loop system. Indeed we can write:

$$
\dot{\tilde{V}}_{D C}=\left[\begin{array}{c}
\dot{\alpha}_{7} \\
\dot{e}_{V_{D C}}
\end{array}\right]=\left[\begin{array}{cc}
0 & K_{7}^{\alpha} \\
-\bar{K}_{7} & -K_{7}
\end{array}\right] \widetilde{V}_{D C}
$$

which closed loop (linear) dynamics is here defined with the eigenvalues:

$$
\lambda_{7 . \alpha_{7}}=-\frac{1}{2}\left(K_{7} \pm \sqrt{\left(K_{7}^{2}-4 \bar{K}_{7} K_{7}^{\alpha}\right)}\right)
$$

As a corollary, there exists $P_{7}=P_{7}^{T}>0$ such that $P_{7} A_{7}+$ $A_{7}^{T} P_{7}=-I_{2}$ where $I_{2}$ is the identity matrix in $\mathbb{R}^{2}$.

We introduce now the Lyapunov function:

$$
V_{7}=\left[\alpha_{7} e_{V_{D C}}\right]^{T} P_{7}\left[\alpha_{7} e_{V_{D C}}\right]
$$

It's derivative is then:

$$
\dot{V}_{7}=-e_{V_{D C}}^{2}-\alpha_{7}^{2}
$$

Now using the property (see [20]) :

$$
\lambda_{\min }\left(P_{7}\right)\|x\|^{2} \leq x^{T} P_{7} x \leq \lambda_{\max }\left(P_{7}\right)\|x\|^{2}
$$

we can then write that $\left(m_{7}\right.$ is a positive constant):

$$
\dot{V}_{7} \leq-\frac{1}{\lambda_{\max }\left(P_{7}\right)} V_{7} \triangleq-m_{7} V_{7}
$$

that implies that:

$$
V_{7}(t)=e^{-m_{7} t} V_{7}(0)
$$

and then we can conclude that:

$$
\lim _{t \rightarrow \infty}\left\|\left[\begin{array}{c}
e_{V_{D C}}(t) \\
\alpha_{7}(t)
\end{array}\right]\right\|=0
$$

and that the closed-loop (linear) dynamics of $V_{D C}$ are exponentially stable.

\section{Control Parameters' tuning:}

The procedure for choosing the parameters of the closed loop system is straightforward and can be found in classical control books as [21]. A simple way for that can be obtained firstly by remarking that the system's desired (closed loop) transfer function can be written as (see [21], [22]):

With $k_{7}=\frac{1}{\omega_{7}^{2}}$.

$$
H_{7}(s)=k_{7} \frac{\omega_{7}^{2}}{s^{2}+2 \zeta_{7} \omega_{7} s+\omega_{7}^{2}}=\frac{1}{s^{2}+K_{7} s+\bar{K}_{7} K_{7}^{\alpha}}
$$

${ }^{1}$ Such matrix always exist because of the stable poles assigned by the tuning gains. we can set $K_{7}^{\alpha}=1$ and then use a simple identification to set $K_{7}$ and $\bar{K}_{7}$ parameters:

$$
\begin{gathered}
\zeta_{7}=\frac{K_{7}}{2 \sqrt{\bar{K}_{7} K_{7}^{\alpha}}} \\
\omega_{7}=\sqrt{\bar{K}_{7} K_{7}^{\alpha}}
\end{gathered}
$$

Aiming at dissociate the current controller and the voltage controller, it was separated the time constants of the controllers; there was imposed a ratio of 100 between $\tau_{\text {icurrent }}$ and $\tau_{\text {ivoltage }}$ (that is true experimentally: the current variations are really quicker than the voltage variations). As a consequence, it was chosen $\omega_{7}=\frac{2 \pi}{T}=\frac{2 \pi}{0.1}=62.831 \mathrm{rad} / \mathrm{s}$. In the same way, it was chosen $\zeta_{7}=0.7$ since it provides a good compromise of quick response without overshoot.

Now, this reference (7) in its turn is split into a Fast and Slow terms by a low pass filter shown in Fig. 2.

$$
i_{S T}^{*}=i_{L 2}^{*}+i_{L 3}^{*}
$$

and then $i_{L 2}^{*}$ provided to the battery acts to maintain the balance of power and $i_{L 3}^{*}$ provided to the supercapacitor acts to dampen fast transitory and oscillations.

2) Current Control for the Battery: we need to design a control law $u_{2}$ such as to steer $i_{L 2}$ towards its reference $i_{L 2}^{*}$.

From system (1):

$$
\dot{i}_{L 2}=\frac{1}{L_{2}} V_{C 2}-\frac{\left[\left(R_{03}-R_{04}\right) u_{2}+R_{04}\right] i_{L 2}}{L_{2}}-\frac{\left(1-u_{2}\right)}{L_{2}} V_{D C}
$$

the tracking error is defined as:

$$
e_{i_{L 2}}=i_{L 2}-i_{L 2}^{*}
$$

Following the backstepping technique, the desired dynamics for $i_{L 2}$ is introduced as:

$$
\begin{aligned}
\dot{\alpha}_{4} & =K_{4}^{\alpha} e_{i_{L 2}} \\
\dot{e}_{i_{L 2}} & =-K_{4} e_{i_{L 2}}-\overline{K_{4}} \alpha_{4}
\end{aligned}
$$

where $\alpha_{4}$ is defined as an extended state (integral term to avoid steady state errors), and positive tuning gain parameters $K_{4}, \bar{K}_{4}$ and $K_{4}^{\alpha}$ that assign the desired dynamics.

From equations (14) and (16) and as in the previous step we obtain the following control law that attain the desired closed loop dynamics:

$$
\begin{aligned}
u_{2}= & \frac{1}{\left(V_{D C}+\left(R_{04}-R_{03}\right) i_{L 2}\right.}\left[V_{D C}-V_{C 2}+R_{04} i_{L 2}\right. \\
& \left.-L_{2}\left(K_{4} e_{i_{L 2}}+\bar{K}_{4} \alpha_{4}-i_{L 2}^{*}\right)\right]
\end{aligned}
$$

With condition $V_{D C}+\left(R_{04}-R_{03}\right) i_{L 2} \neq 0$ (which is always true by physical reasons). The same procedure is repeated to tune control parameters for the current control loop of the battery: $K_{4}=8796.2, \bar{K}_{4}=6283^{2}$ and $K_{4}^{\alpha}=1$ 
3) Supercapacitor's controller: The control input $u_{3}$ is dedicated to control the dynamics $i_{L 3}$ to ensure a desired charge/discharge of the supercapacitor. From system (1):

$\frac{d i_{L 3}}{d t}=\frac{1}{L_{3}} V_{C 3}-\frac{\left[\left(R_{05}-R_{06}\right) u_{3}+R_{06}\right] i_{L 3}}{L_{3}}-\frac{\left(1-u_{3}\right)}{L_{3}} V_{D C}$

The tracking error is defined as:

$$
e_{i_{L 3}}=i_{L 3}-i_{L 3}^{*}
$$

following the backstepping technique, the desired dynamics for $i_{L 3}$ is introduced as:

$$
\begin{aligned}
\dot{\alpha}_{6} & =K_{6}^{\alpha} e_{i_{L 3}} \\
\dot{e}_{i_{L 3}} & =-K_{6} e_{i_{L 3}}-\overline{K_{6}} \alpha_{6}
\end{aligned}
$$

From equations (18) and (20) and with the positive tuning gain parameters $K_{6}, K_{6}^{\alpha}$ and $\bar{K}_{6}$, we obtain the control law:

$$
\begin{aligned}
u_{3}= & \frac{1}{\left(V_{D C}+\left(R_{06}-R_{05}\right) i_{L 3}\right.}\left[V_{D C}-V_{C 3}\right. \\
& \left.+R_{06} i_{L 3}-L_{3}\left(K_{6} e_{i_{L 3}}+\bar{K}_{6} \alpha_{6}-\dot{i}_{L 3}^{*}\right)\right]
\end{aligned}
$$

with condition $V_{D C}+\left(R_{06}-R_{05}\right) i_{L 3} \neq 0$ (again always true by physical reasons). The same procedure is repeated to tune the control parameters: $K_{6}=87963.4, \bar{K}_{6}=62831^{2}$ and $K_{6}^{\alpha}=1$.

We introduce now the Lyapunov function where $P_{4,6}$ fulfill the Lyapunov's equation:

$$
V_{4,6}=\left(\left[\begin{array}{ll}
\alpha_{4} & e_{i_{L 2}}
\end{array}\right]^{T} P_{4}\left[\begin{array}{ll}
\alpha_{4} & e_{i_{L 2}}
\end{array}\right]\right)+\left(\left[\begin{array}{ll}
\alpha_{6} & e_{i_{L 3}}
\end{array}\right]^{T} P_{6}\left[\begin{array}{ll}
\alpha_{6} & e_{i_{L 3}}
\end{array}\right]\right)
$$

It's derivative is then:

$$
\dot{V}_{4,6}=-e_{i_{L 2}}^{2}-\alpha_{4}^{2}-e_{i_{L 3}}^{2}-\alpha_{6}^{2}
$$

which implies that the closed loop system for the battery and supercapacitor is exponentially stable.

\section{B. Control law for $P V$}

Let us first focus on the control $u_{1}$ dedicated to stabilize dynamics $V_{C 1}$ and $i_{L 1}$ : it is developed by using a backstepping technique such as to design an $i_{L 1}^{*}$ reference value to $i_{L 1}$ that is used to steer voltage $V_{C 1}$ to its equilibrium point following a desired dynamic behavior to be imposed. From system (1):

$$
\begin{aligned}
\frac{d V_{C 1}}{d t} & =-\frac{1}{R_{1} C_{1}} V_{C 1}-\frac{1}{C_{1}} i_{L 1}+\frac{1}{R_{1} C_{1}} V_{P V} \\
\frac{d i_{L 1}}{d t} & =\frac{1}{L_{1}} V_{C 1}-\frac{\left[\left(R_{01}-R_{02}\right) u_{1}+R_{02}\right] i_{L 1}}{L_{1}}-\frac{\left(1-u_{1}\right)}{L_{1}} V_{D C}
\end{aligned}
$$

Lets first define the output tracking errors:

$$
\begin{array}{r}
e_{V_{C 1}}=\left(V_{C 1}-V_{C 1}^{*}\right) \\
e_{i_{L 1}}=\left(i_{L 1}-i_{L 1}^{*}\right)
\end{array}
$$

The desired dynamics for $V_{C 1}$ and $i_{L 1}$ are introduced as:

$$
\dot{e}_{V_{C 1}}=-K_{1} e_{V_{C 1}}-\overline{K_{1}} \alpha_{1}
$$

$$
\dot{e}_{i_{L 1}}=-K_{2} e_{i_{L 1}}-\overline{K_{2}} \alpha_{2}
$$

where $\alpha_{1}$ and $\alpha_{2}$ represent the integral terms assuring zero error in steady state between the dynamics of $V_{C 1}$ and $i_{L 1}$ :

$$
\begin{aligned}
& \dot{\alpha}_{1}=K_{1}^{\alpha} e_{V_{C 1}} \\
& \dot{\alpha}_{2}=K_{2}^{\alpha} e_{i_{L 1}}
\end{aligned}
$$

and $K_{1}, \bar{K}_{1}, K_{1}^{\alpha}, K_{2}, \bar{K}_{2}$ and $K_{2}^{\alpha}$ are positive tuning gain parameters that are used to impose desired dynamics to the closed loop system. From equations (24), (25) and (27) we can then design:

$$
i_{L 1}^{*}=\frac{1}{R_{1}}\left(V_{P V}-V_{C 1}\right)+C_{1} K_{1}\left(V_{C 1}-V_{C 1}^{*}\right)+C_{1} \bar{K}_{1} \alpha_{1}
$$

that feedback linearizes the first equation of (1). From equations (24), (26) and (28) we can propose the control law:

$$
\begin{aligned}
u_{1}= & \frac{1}{V_{D C}+\left(R_{02}-R_{01}\right) i_{L 1}}\left[V_{D C}-V_{C 1}+R_{02} i_{L 1}\right. \\
& -L_{1}\left(K_{2}\left(i_{L 1}-i_{L 1}^{*}\right)+\bar{K}_{2} \alpha_{2}-C_{1} \bar{K}_{1} K_{1}^{\alpha} e_{V_{C 1}}\right. \\
& \left.\left.+\left(C_{1} K_{1}-\frac{1}{R_{1}}\right)\left(K_{1} e_{V_{C 1}}+\bar{K}_{1} \alpha_{1}\right)\right)\right]
\end{aligned}
$$

with the condition (always fulfilled in reality):

$$
V_{D C}+\left(R_{02}-R_{01}\right) i_{L 1} \neq 0
$$

The control input $u_{1}$ in its turn, feedback linearizes the dynamics of $V_{C 1}$ and $i_{L 1}$. We can then introduce the Lyapunov function:

$$
V_{1,2}=\left(\left[\begin{array}{ll}
\alpha_{1} & e_{V_{C 1}}
\end{array}\right]^{T} P_{1}\left[\alpha_{1} e_{V_{C 1}}\right]\right)+\left(\left[\begin{array}{ll}
\alpha_{2} & e_{i_{L 1}}
\end{array}\right]^{T} P_{2}\left[\begin{array}{ll}
\alpha_{2} & e_{i_{L 1}}
\end{array}\right]\right)
$$

where matrices $P_{1.2}$ satisfy the Lyapunov equation. The Lyapunov's function derivative is then:

$$
\dot{V}_{1.2}=-e_{V_{C 1}}^{2}-\alpha_{1}^{2}-e_{i_{L 1}}^{2}-\alpha_{2}^{2}
$$

and then we can conclude that the closed-loop dynamics of $V_{C 1}$ and $i_{L 1}$ are exponentially stable.

\section{StABILITY StUdy FOR THE INTERCONNECTED SYSTEM}

It is possible to split the states into controlled variables $V_{C 1}, i_{L 1}, i_{L 2}, i_{L 3}$ and $V_{D C}$ and the zero dynamics (uncontrolled variables) $V_{C 2}$ and $V_{C 3}$ :

$$
\left[\begin{array}{c}
V_{C 1} \\
i_{L 1} \\
V_{C 2} \\
i_{L 2} \\
V_{C 3} \\
i_{L 3} \\
V_{D C}
\end{array}\right]=\left[\begin{array}{c}
\text { Controlled dynamics } \\
\text { Controlled dynamics } \\
\text { Zero dynamics } \\
\text { Controlled dynamics } \\
\text { Zero dynamics } \\
\text { Controlled dynamics } \\
\text { Controlled dynamics }
\end{array}\right]
$$

Let us now move the focus to the zero dynamics. We can rewrite the zero dynamics equations of system (1) as: 


$$
\begin{aligned}
\frac{d V_{C 2}}{d t} & =-\frac{1}{R_{2} C_{2}} V_{C 2}-\frac{1}{C_{2}} i_{L 2}+\frac{1}{R_{2} C_{2}} V_{B A T} \\
\frac{d V_{C 3}}{d t} & =-\frac{1}{R_{3} C_{3}} V_{C 3}-\frac{1}{C_{3}} i_{L 3}+\frac{1}{R_{3} C_{3}} V_{S C}
\end{aligned}
$$

and we can compute $V_{C 2}^{e}$ and $V_{C 3}^{e}$ their equilibrium points:

$$
\begin{aligned}
0 & =-\frac{1}{R_{2} C_{2}} V_{C 2}-\frac{1}{C_{2}} i_{L 2}^{*}+\frac{1}{R_{2} C_{2}} V_{B A T} \\
0 & =-\frac{1}{R_{3} C_{3}} V_{C 3}-\frac{1}{C_{3}} i_{L 3}^{*}+\frac{1}{R_{3} C_{3}} V_{S C}
\end{aligned}
$$

and then define the complete equilibrium vector:

$$
x^{e}=\left[\begin{array}{c}
V_{C 1}^{*} \\
i_{L 1}^{*} \\
V_{C 2}^{e} \\
i_{L 2}^{*} \\
V_{C 3}^{e} \\
i_{L 3}^{*} \\
V_{D C}^{*}
\end{array}\right]
$$

We define then the errors $e_{V_{C 2}}$ and $e_{V_{C 3}}$ as:

$$
\begin{aligned}
& e_{V_{C 2}}=\left(V_{C 2}-V_{C 2}^{e}\right) \\
& e_{V_{C 3}}=\left(V_{C 3}-V_{C 3}^{e}\right)
\end{aligned}
$$

The Lyapunov candidate function $V_{3,5}$ is then:

$$
V_{3,5}=\frac{R_{2} C_{2}}{2} e_{V_{C 2}}^{2}+\frac{R_{3} C_{3}}{2} e_{V_{C 3}}^{2}
$$

which time derivative is:

$$
\dot{V}_{3,5}=-e_{V_{C 2}}^{2}-e_{V_{C 3}}^{2}
$$

that again, assures exponential stability of the zero dynamics.

We can now regroup these partial results in the form of the following theorem that establish the overall stability property.

Theorem V.1. : Consider system (1) and its equilibrium points set (37). Under the assumption that for each time the conditions:

$$
\begin{aligned}
& V_{D C}+\left(R_{02}-R_{01}\right) i_{L 1} \neq 0 \\
& V_{D C}+\left(R_{04}-R_{03}\right) i_{L 2} \neq 0 \\
& V_{D C}+\left(R_{06}-R_{05}\right) i_{L 3} \neq 0
\end{aligned}
$$

hold, then there exist control laws $u_{1}, u_{2}$ and $u_{3}$ given respectively by (30), (17) and (21); positive design parameters $K_{1}$, $\bar{K}_{1}, K_{1}^{\alpha}, K_{2}, \bar{K}_{2}, K_{2}^{\alpha}, K_{4}, \bar{K}_{4}, K_{4}^{\alpha}, K_{6}, \bar{K}_{6}$ and $K_{6}^{\alpha}, K_{7}$, $\bar{K}_{7}$ and $K_{7}^{\alpha}$, such that the closed loop system is exponentially stable.

Proof. We can finally state the constructive Lyapunov function for the whole system $V_{1,2,3,4,5,6,7}=V_{1}+V_{2}+V_{3}+V_{4}+V_{5}+$ $V_{6}+V_{7}$ as follows:

$$
\begin{aligned}
& V_{1} \ldots 7=\left(\left[\alpha_{1} e_{V_{C 1}}\right]^{T} P_{1}\left[\alpha_{1} e_{V_{D C}}\right]\right)+\left(\left[\alpha_{2} e_{i_{L 1}}\right]^{T} P_{2}\left[\alpha_{2} e_{i_{L 1}}\right]\right) \\
& +\frac{R_{2} C_{2}}{2} e_{V_{C 2}}^{2}+\left(\left[\alpha_{4} e_{i_{L 2}}\right]^{T} P_{4}\left[\alpha_{4} e_{i_{L 2}}\right]\right)+\frac{R_{3} C_{3}}{2} e_{V_{C 3}}^{2} \\
& +\left(\left[\alpha_{6} e_{i_{L 3}}\right]^{T} P_{6}\left[\alpha_{6} e_{i_{L 3}}\right]\right)+\left(\left[\alpha_{7} e_{V_{D C}}\right]^{T} P_{7}\left[\alpha_{7} e_{V_{D C}}\right]\right)
\end{aligned}
$$

It's derivative is then:

$$
\begin{aligned}
& \dot{V}_{1 \ldots 7}=-e_{V_{C 1}}^{2}-\alpha_{1}^{2}-e_{i_{L 1}}^{2}-\alpha_{2}^{2}-e_{V_{C 2}}^{2}-e_{i_{L 2}}^{2}-\alpha_{4}^{2}-e_{V_{C 3}}^{2} \\
& -e_{i_{L 3}}^{2}-\alpha_{6}^{2}-e_{V_{D C}}^{2}-\alpha_{7}^{2}-e_{V_{C 2}} e_{i_{L 2}}-e_{V_{C 3}} e_{i_{L 3}}
\end{aligned}
$$

that completing the squares can be rewritten as:

$$
\begin{aligned}
& \dot{V}_{1 \ldots 7} \leq-e_{V_{C 1}}^{2}-\alpha_{1}^{2}-e_{i_{L 1}}^{2}-\alpha_{2}^{2}-\alpha_{4}^{2}-\alpha_{6}^{2}-e_{V_{D C}}^{2}-\alpha_{7}^{2} \\
& +\left(1-\frac{1}{\sqrt{2}}\right)\left(-e_{V_{C 2}}^{2}-e_{i_{L 2}}^{2}-e_{V_{C 3}}^{2}-e_{i_{L 3}}^{2}\right)
\end{aligned}
$$

This in its turn again, assures the exponential convergence of all states of the interconnected system towards their equilibrium points.

\section{Simulation Result}

MATLAB/Simulink is used for simulation studies of a DC microgrid consisting of solar, battery and supercapacitor units supplying a variable resistive load. The system configuration is as shown in Figure (1) and the parameters were discussed in Section IV. The model parameters' values are depicted in Table (I).

\begin{tabular}{|l|r|}
\hline$R_{02}, R_{04}, R_{06}$ & $0.045 \Omega$ \\
\hline$R_{01}, R_{03}, R_{05}$ & $0.044 \Omega$ \\
\hline$R_{1}, R_{2}, R_{3}$ & $0.14 \Omega$ \\
\hline$C_{1}, C_{2}, C_{3}$ & $4700 e^{-6} \mathrm{~F}$ \\
\hline$C_{D C}$ & $1500 e^{-6} \mathrm{~F}$ \\
\hline$L_{1}, L_{2}, L_{3}$ & $100 e^{-6} \mathrm{H}$ \\
\hline$V_{D C}$ & $50 \mathrm{~V}$ \\
\hline
\end{tabular}

Table I: DC MicroGrid parameters

The main goal is to demonstrate that the proposed control strategy provides good results for cases of varying solar irradiance as well as load variations. It is expected to regulate the DC bus voltage with battery and supercapacitor units in such a way that the supercapacitor provides the fast changing components of the required DC bus voltage compensating current while the battery unit provides the slow varying components. This is achieved with a signal decomposition block with a $20 \mathrm{~Hz}$ LPF. As in the section of experimental results, since the voltages in the SC and battery did not vary much, their waveforms are not shown.

Figure (4) shows how the DC bus voltage (top) varies as the load current and PV current (following screens) vary. The load impedance is equal to $10,4 \Omega$ for $0.06 \mathrm{~s}<t<0.65 \mathrm{~s}$ and is equal to $21 \Omega$ elsewhere. The solar irradiance was the rated one for $t<0.22 \mathrm{~s}$ and $t>0.46 \mathrm{~s}$, and equal to $200 \mathrm{~W} / \mathrm{m}^{2}$ otherwise. There, one sees that the DC bus voltage varies briefly following the transients, quickly recovering and stabilizing at the rated $(50 \mathrm{~V}) \mathrm{DC}$ bus voltage in about $1 \mathrm{~ms}$. The maximum DC bus voltage error is equal to $0.08 \mathrm{~V}$.

This is achieved by the action of the proposed control scheme making use of the battery and supercapacitor interfaces. The reference and actual waveforms of the former are shown in the fourth screen from the top. The reference signal varies slowly and the actual waveform follows it with a small switching frequency ripple. Besides, the battery current is the one required to balance the power in the DC microgrid in 


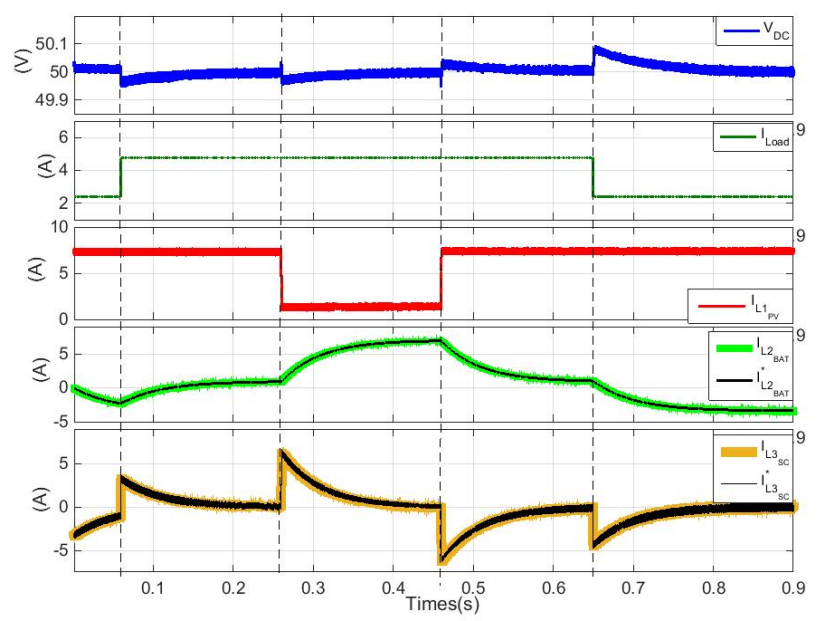

Figure 4: Dynamic response of the system

steady-state.

The reference and actual waveforms of the supercapacitor unit are shown in the fifth screen from the top. This reference varies fast, following PV generation and load demand variations, returning to $0 A$ after transitory.

These results confirm that the proposed scheme is effective in achieving the goals defined for this application.

\section{EXPERIMENTAL VALIDATION}

A DC MicroGrid with a RES, two energy storage units and a variable load was assembled in the laboratory to verify the performance of the proposed control scheme experimentally. An Agilent Solar Array Simulator $(S A S)(E 4350 B)$ was used to emulate a solar array. It was programmed to provide a maximum power of $213 \mathrm{~W}$ with $7.35 \mathrm{~A}$ and $29 \mathrm{~V}$ at rated solar irradiance.

Maxwell supercapacitor modules (BMOD0165P048) of $165 \mathrm{~F}$ with a rated voltage of $48 \mathrm{~V}$ were employed for the energy storage units. One is used as a battery and the second is used as a supercapacitor.

Regarding the power electronics interface, a Semikron MiniSKiiP8 Three-phase $1200 \mathrm{~V}$ Power board, with a MiniSKiiP $83 A C$ power module and a $S K H I 61$ IGBT driver, was used in this study. One leg of the three-phase inverter is used for each DER. An LC filter $(100 \mu H$ and $470 \mu F)$ is connected between the mid-points of the three-phase inverter legs and the storage and the source elements, to create the classical bi-directional Class C DC-DC converters. A $1500 \mu F$ capacitor at the DC bus of the inverter corresponds to the main filter at the DC bus of the MicroGrid.

The converters switch at $20 \mathrm{kHz}$. A $20 \mu \mathrm{s}$ time step is used in this case. The reference voltage for the DC bus is kept at $50 \mathrm{~V}$. A set of ten parallel switchable $44 \Omega$ resistors were used for realizing a variable load, the battery is in discharge mode. A picture of the experimental set-up is shown in the following Figure.

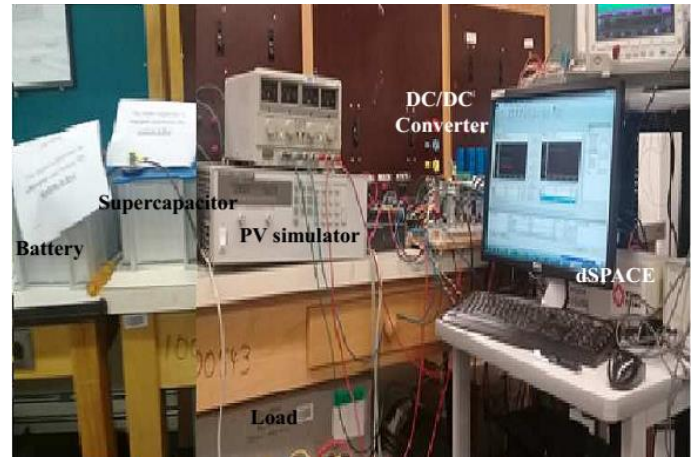

Figure 5: Experimental setup

The main objective of the tests is to observe the performance of the system, mostly the regulation of the DC bus voltage and the split of the required compensating current among the supercapacitor and battery converter units. In this study, the cut-off frequency of the LPF is equal to $20 \mathrm{~Hz}$, as in the simulations. The voltages in the supercapacitor and battery are equal to $24 \mathrm{~V}$ and $28 \mathrm{~V}$, respectively. Considering the short time frames addressed in the performance results shown in this study, there should be no noticeable change in their voltage magnitudes, which are not shown in the results presented below.

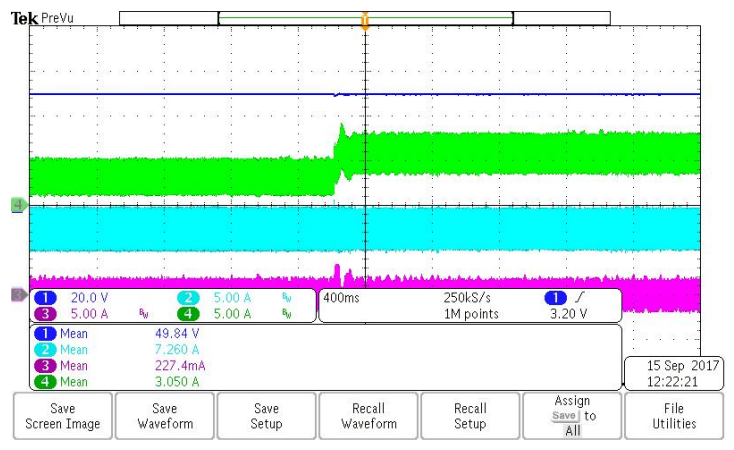

Figure 6: Screen shot of the scope

Figure (6) shows a screen shot of the scope with the response of the system (DC bus voltage in blue, battery current in green, PV current in light blue and supercapacitor current in pink) to an increase in the power demand, when the load is changed from $8.8 \Omega$ (five resistors of $44 \Omega$ in parallel) to $7.35 \Omega$ (six resistors of $44 \Omega$ in parallel). Since it is difficult to see the details of the waveforms, the stored data was sent to MATLAB where it is easier to rescale the waveforms emphasizing their key features for performance analysis.

This is what is shown in Figure (7). In such a case, the load changed at $t=1 \mathrm{~s}$. On the top, one sees that the DC bus is initially equal to $50 \mathrm{~V}$, decreasing briefly by about $0.9 \mathrm{~V}$ following the load step, then returning, in about $0.15 \mathrm{~s}$, towards the original value. In the next plot, the current in the inductor of the PV interface remains virtually unaffected at the MPPT value. Just below, one sees the inductor currents of the battery and supercapacitor interfaces. The former initially provides about $3.1 A$, increasing its output rather slowly, to meet the average value of the step load increase at $t=1 \mathrm{~s}$. Conversely, in the very bottom, the inductor current of the supercapacitor 


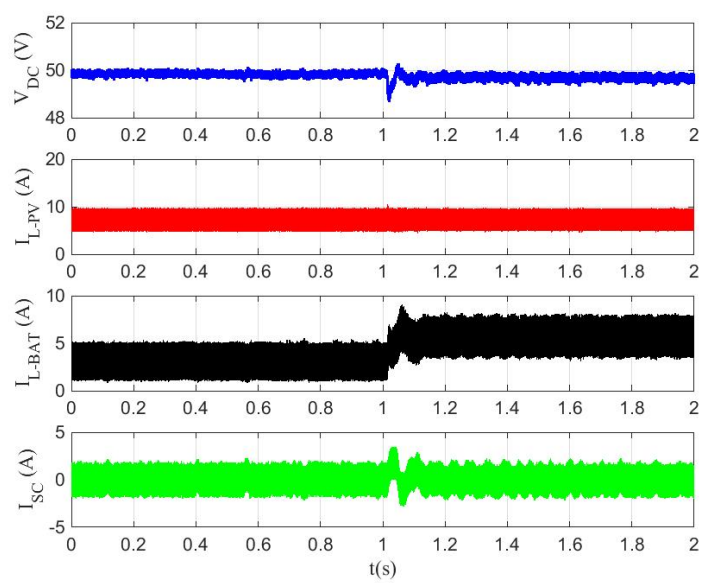

Figure 7: Dynamic Response during increasing load with battery discharging

interface starts at $0 A$, showing that it is not providing any average power. Following the load increase, the contribution of the supercapacitor converter increases very fast and returns to an average of about $0 \mathrm{~A}$, as the battery interface takes over the power balancing in the system.

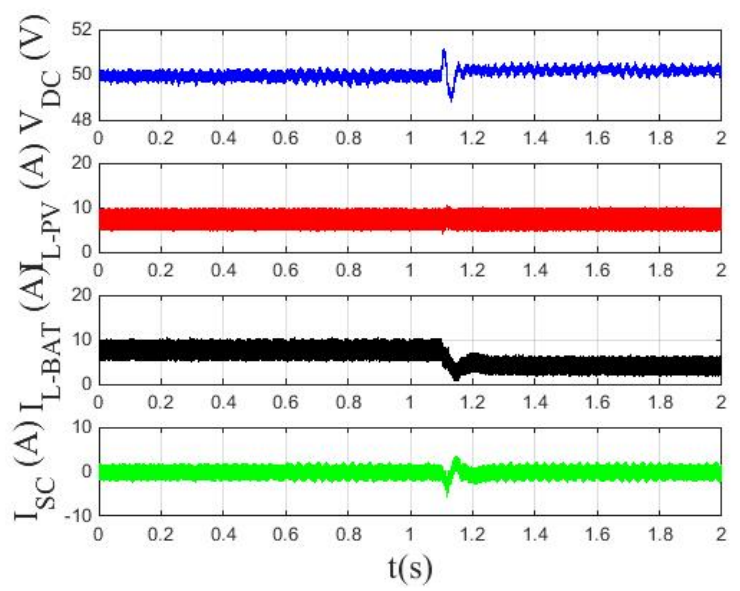

Figure 8: Dynamic Response during deacreasing load with battery discharging

Figure (8) shows the opposite change: A step load decrease at $t=0.11 \mathrm{~s}$. In such a case, the DC bus voltage will initially increase, by about $0.9 \mathrm{~V}$, then return to its original value as the battery and supercapacitor interfaces adjust their injected powers. As before, the supercapacitor interface reacts faster than the battery one, what allows the current in the latter to vary at a lower rate, as desired.

Figure (9) shows a step load decrease at $t=0.6 \mathrm{~s}$. The DC bus voltage increases, by about $0.9 \mathrm{~V}$, then returns to its original value. The supercapacitor absorbs exceeding power for a very short time while battery adjusts surplus of power.

Figure (10) shows a step load increase at $t=0.7 \mathrm{~s}$. The DC bus voltage decreases by about $1.1 \mathrm{~V}$, then returns to its original value as the battery and supercapacitor interfaces adjust their injected powers.

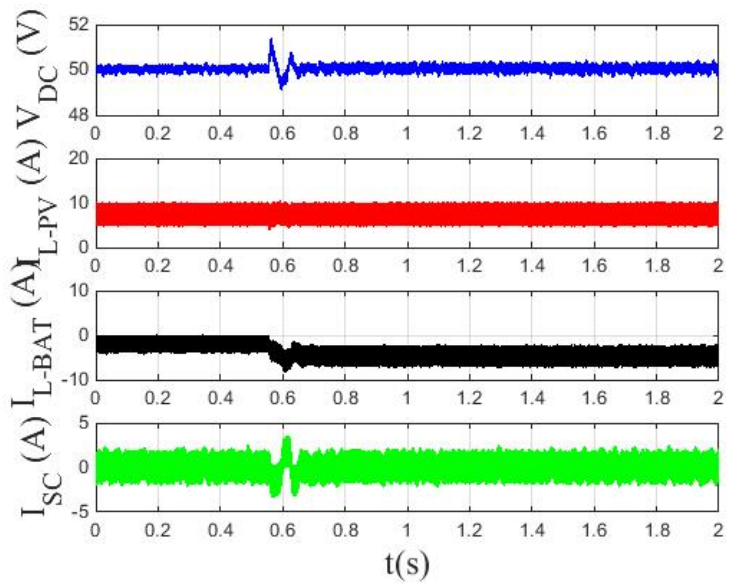

Figure 9: Dynamic Response during decreasing load with battery charging

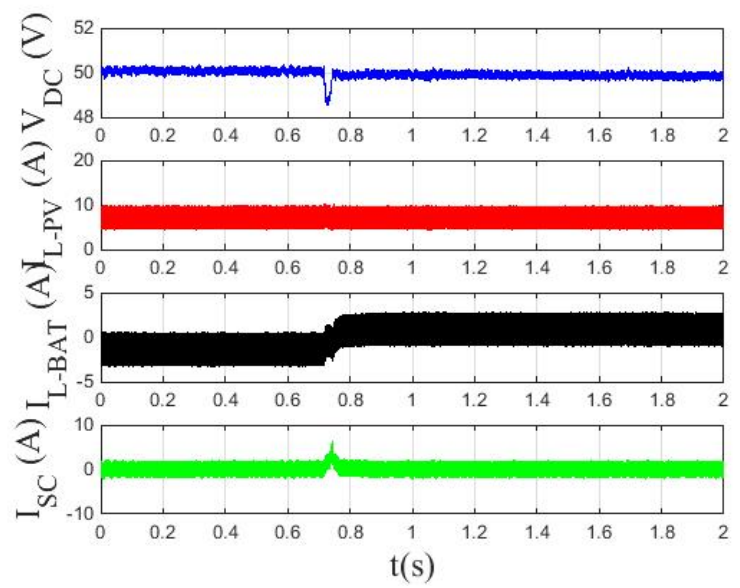

Figure 10: Dynamic Response during increasing load with battery charging

As before, the supercapacitor interface reacts faster than the battery one, what allows the current in the latter to vary at a lower rate, as desired.

\section{Experiments' Results}

The response for step power changes is fast, returning to nominal value of $V_{D C}$ in about $t=0.04 s$. The $V_{D C}$ output has small ripple, showing that the power quality is good, and that we can reduce the size of capacitor in the converter.

\section{COMPARISON WITH PI CONTROL}

In order to demonstrate the performance of the proposed control strategy, it is compared with PI linear control . State space averaging method is used to obtain a Small Signal model for the converter, see ( [23]-[25]). The model is derived under the assumption that all the converter elements are ideal. The PI controllers are designed using method cited in ( [26], [27]).

Figure (11) presents a comparison on DC MicroGrid voltage dynamics between a classical linear PI controller and the proposed nonlinear controller. There one sees that the DC 


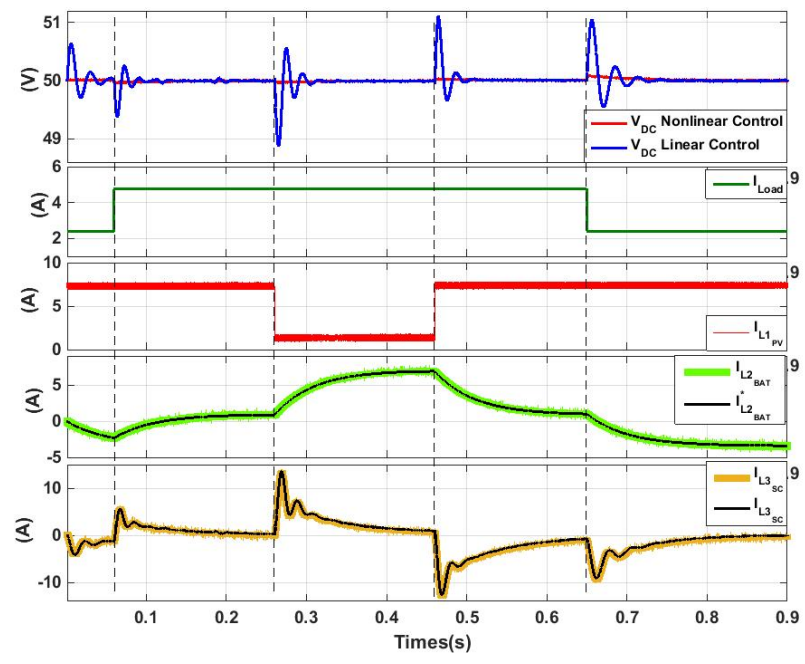

Figure 11: Comparison of the $V_{D C}$ dynamics by applying nonlinear control in red and classical PI control in blue

bus voltage varies following the transients, recovering and stabilizing at the rated $(50 \mathrm{~V})$. The maximum DC bus voltage error is equal to $1.2 \mathrm{~V}$ for linear control in blue.

In the PI linear control, the voltage $V_{D C}$ oscillates for $60 \mathrm{~ms}$ before reaching steady state while the steady-state response time for the proposed nonlinear control is reached at $1 \mathrm{~ms}$ as designed (it could be chosen to be faster though).

For the battery, the reference and actual waveforms are shown in the fourth screen from the top. The reference signal varies slowly and the actual waveform follows it with a small switching frequency ripple.

For the SuperCapacitor, its reference and actual waveforms are shown in the last screen from the top. The reference signal varies fast and the actual waveform follows it, with a big switching frequency ripple, following PV generation, load demand variations, and $V_{D C}$ oscillations, converging to $0 A$ in steady state.

It can be concluded that the PI linear control response have bigger overshoots in transients and a slower response speed.

\section{CONCLUSION}

DC MicroGrids offer an ideal environment for the integration of large amounts of stochastic renewable energy sources. Sudden and large power variations, generation and demand, can be prevented by means of a hybrid (power and energy) storage system. This is usually accomplished with linear type controllers designed based on linearized plant models, for a certain operating condition. As this changes, so do the dynamic responses of key system variables. In such cases, the system stability cannot be assure in the strict sense. As an alternative, this paper has discussed the use of a two level hierarchical control strategy that ensures the stability of all variables, in particular the voltage of the DC bus, in spite of strong and random variations of power generation and demand. The low level controller is based on nonlinear theory and assures exponential stability of each element as well as of the whole system under a wide range of operating conditions. On top of that, a high level controller provides references to accomplish the appropriate power flow among system components. A rigorous stability analysis, that takes into account the whole system by a constructive Lyapunov function, is performed. Unlike conventional linear approaches, the proposed control structure will assign desired dynamics to variables of the DC MicroGrid, and along with a final Lyapunov function, will provide the required controller parameters. It should be noted that the obtained control algorithms are very simple, close to nested PIs with feedforward compensating terms. They can be easily implemented in real applications, without the need of sophisticated digital control hardware. Simulation and experimental results of a DC MicroGrid with a Photovoltaic source, two storage units and a variable load are presented to corroborate the theoretical analysis, proposed control scheme and design procedure. It is shown that the system presents a good dynamic performance under various operating conditions created by strong random variations of power generation and consumption. Future works will address the comparison of the proposed controllers with classical ones, in terms of performance, robustness and required resources.

\section{REFERENCES}

[1] H.Bevrani, B. Francois, T.Ise, Microgrid Dynamics and Control, John Wiley and Sons Inc, 2017.

[2] N. Hatziargyriou, Microgrids: Architectures and Control, John Wiley and Sons, 2014, 2014.

[3] E.Jimenez, M.J.Carrizosa, A.Benchaib, G.Damm, F. L. Lagarrigue, A new generalized power flow method for multi connected dc grids, International Journal of Electrical Power and Energy Systems 74 (2016) 329-337.

[4] A.Sajadi, S.S.Sebtahmadi, M.Koniak, P. Biczel, S. Mekhilef, Distributed control scheme for voltage regulation in smart grids, International Journal of Smart Grid and Clean Energy 1 (2012) 55 - 59.

[5] R. H. Lasseter, Smart distribution: Coupled microgrids, Proceedings of the IEEE 99 (6) (2011) 1074-1082.

[6] S. M. Ashabani, Y. A. r. I. Mohamed, New family of microgrid control and management strategies in smart distribution grids; analysis, comparison and testing, IEEE Transactions on Power Systems 29 (5) (2014) 2257-2269.

[7] E. Rokrok, M. Shafie-Khah, J. P. S. Catalão, Comparison of two control strategies in an autonomous hybrid microgrid, in: 2017 IEEE PES Innovative Smart Grid Technologies Conference Europe (ISGT-Europe), 2017, pp. 1-6.

[8] S. K. Kollimalla, M. K. Mishra, N. L. Narasamma, Design and analysis of novel control strategy for battery and supercapacitor storage system, IEEE Transactions on Sustainable Energy 5 (4) (2014) 1137-1144.

[9] J. Dulout, B. Jammes, L. Seguier, C. Alonso, Control and design of a hybrid energy storage system, in: 2015 17th European Conference on Power Electronics and Applications (EPE 15 ECCE-Europe), 2015, pp. $1-9$.

[10] B. Liu, F. Zhuo, Y. Zhu, H. Yi, System operation and energy management of a renewable energy-based dc micro-grid for high penetration depth application, IEEE Transactions on Smart Grid 6 (3) (2015) 1147 1155.

[11] A. Benchaib, Advanced Control of AC / DC Power Networks: System of Systems Approach Based on Spatio-temporal Scales, Wiley-ISTE, 2015.

[12] P. Kundur, J. Paserba, V. Ajjarapu, G. Andersson, A. Bose, C. Canizares, N. Hatziargyriou, D. Hill, A. Stankovic, C. Taylor, T. V. Cutsem, V. Vittal, Definition and classification of power system stability $I E E E / C I G R E$ joint task force on stability terms and definitions, IEEE Transactions on Power Systems 19 (3) (2004) 1387-1401.

[13] S. Siad, G. Damm, L. G. Dol, A. d. Bernardinis, Design and control of a dc grid for railway stations, in: PCIM Europe 2017, International Exhibition and Conference for Power Electronics, Intelligent Motion, Renewable Energy and Energy Management, 2017, pp. 1-8. 
[14] Y. Chen, G. Damm, A. Benchaib, M. Netto, F. Lamnabhi-Lagarrigue, Control induced explicit time-scale separation to attain dc voltage stability for a vsc-hvdc terminal, IFAC Proceedings Volumes 47 (3) (2014) 540 - 545, 19th IFAC World Congress.

[15] A. Iovine, S. Benamane Siad, G. Damm, E. De Santis, M. D Di Benedetto, Nonlinear control of a dc microgrid for the integration of photovoltaic panels, IEEE Transactions on Automation Science and Engineering 14 (2) (2017) 524-535.

[16] Merdassi.A, La modellisation Automatique pour l'electronique de puissance, Editions Universitaires Europeennes, 2010.

[17] R. S. Bhatia, B. Singh, D. K. Jain, S. P. Jain, Battery energy storage system based power conditioner for improved performance of hybrid power generation, in: 2008 Joint International Conference on Power System Technology and IEEE Power India Conference, 2008, pp. 16.

[18] S. R. Sanders, J. M. Noworolski, X. Z. Liu, G. C. Verghese, Generalized averaging method for power conversion circuits, IEEE Transactions on Power Electronics 6 (2) (1991) 251-259.

[19] J. Lee, B. Han, N. Choi, Dc micro-grid operational analysis with detailed simulation model for distributed generation, in: 2010 IEEE Energy Conversion Congress and Exposition, 2010, pp. 3153-3160.
[20] H. Khalil, Nonlinear Systems, Pearson; Edition, 2002.

[21] K. Ogata, Modern Control Engineering, 5th Edition, Pearson, 2010.

[22] H. Bourles., Linear Systems, ISTE Ltd and John Wiley and Sons, 2010.

[23] Y. Jin, J. Xu, G. Zhou, C. Mi, Small-signal modeling and analysis of improved digital peak current control of boost converter, in: 2009 IEEE 6th International Power Electronics and Motion Control Conference, 2009, pp. 326-330.

[24] W. Tang, F. C. Lee, R. B. Ridley, Small-signal modeling of average current-mode control, IEEE Transactions on Power Electronics 8 (2) (1993) 112-119.

[25] J. Sun, R. M. Bass, Modeling and practical design issues for average current control, in: APEC '99. Fourteenth Annual Applied Power Electronics Conference and Exposition. 1999 Conference Proceedings, Vol. 2, 1999, pp. 980-986.

[26] S. M. Cherati, N. A. Azli, S. M. Ayob, A. Mortezaei, Design of a current mode pi controller for a single-phase pwm inverter, in: 2011 IEEE Applied Power Electronics Colloquium (IAPEC), 2011, pp. 180184.

[27] L. H. Dixon, Average Current Mode Control of Switching Power Supplies,Unitrode Power Supply Design Seminar Manual, 1990. 